\title{
Development of CMOS Image Sensor System based on Chaos Particle Swarm
}

\author{
Hongyu Duan ${ }^{1 *}$ and Fengxia Yang ${ }^{2}$ \\ ${ }^{1}$ Department of Information Engineering, Henan University of Animal Husbandry and \\ Economy, Henan Zhengzhou 450011, China \\ ${ }^{2}$ Department of Information Engineering, Henan College of Finance \& taxation, \\ Henan Zhengzhou 450011, China \\ *Corresponding Author: duanhongyuzz@163.com
}

\begin{abstract}
Particle swarm optimization algorithm in solving complex functions, such as slow convergence, accuracy is not high, easily falling into local optimum problem. Based on the chaos optimization is introduced into particle swarm optimization algorithm, given the chaotic particle swarm optimization algorithm. In order to improve the image quality of CMOS image sensor, the image of the main noise source and the distortion of image analysis, this paper present the development of CMOS image sensor system based on chaos particle swarm. Computer CMOS image sensor image quality simulation, the results show that chaotic particle swarm optimization algorithm convergence performance is better than that of particle swarm optimization algorithm.
\end{abstract}

Keywords: Chaos, CMOS Image Sensor, Particle Swarm

\section{Introduction}

Particle Swarm Optimization algorithm is simple, robust good number of advantages, but evolutionary slow late convergence, easy to fall into local minimum. Can take some kind of optimization methods to further improve the quality of each generation groups, will undoubtedly contribute to the subsequent search process. Chaos optimization the chaos optimization has been widely used in recent years [1]. It chaotic variable mapping optimization problem model to take full advantage of the chaos variables in the chaotic motion process periodicity, randomness, regularity to find the globally optimal solution.

Chaos is a ubiquitous nonlinear phenomenon, non-deterministic random motion state determined by the equation. Randomness, periodicity and regularity of chaotic variables, the chaotic optimization introduces the idea of the particle swarm algorithm; prevent certain particles Diego stagnation in recent years on behalf of, so as to solve the problem of prematurely.

PSO algorithm iteration no change in the current structure of the particle swarm, the particles continuing ability to search, improved algorithm to keep the PSO arithmetic and simple structure. Introducing chaotic mutation have a wider distribution of the particles mutated, and thus able to coarse-grained global search, which helps the particle to jump out early into a local optimum acceleration to find the area where most advantages. area near the optimal value gradually with the increase in the number of iterations, the groups most advantages, this time particle should not be too much deviation from the original location to avoid blind search within the large space, but also to ensure the accuracy of the search can be 
seen, the particle swarm optimization algorithm with chaotic fusion, combining the advantages of both, each other learn from each other, chaos particle swarm optimization algorithm than the basic particle swarm optimization algorithm to optimize performance.

Image acquisition and monitor the most critical part of the video chain, this is because the image signal processing capabilities for effective information extracted from the video stream is closely related to the quality of the acquired image, perhaps seemingly esoteric, in fact, the essence is the sensor quality and a combination of the quality of the sensor output processing. The trends of the security monitoring system is image processing functions to migrate to the edge of the network - that is, to move the camera inside, close to the sensor. Flexibility and efficiency is the highest goal of the product development cycle. This paper discusses the integration of CMOS image sensors, microcontrollers and signal processing unit within the FPGA.

In order to improve the image quality of a CMOS image sensor, through the analysis of the image the main source of noise and image distortion, the paper proposes a novel CMOS active pixel image sensor. The CMOS image sensor uses a 4T active pixel, greatly improving the sensitivity of the image sensor. Through the image preprocessing functions integrated in the sensor, to improve the quality of the image play a very good effect. The image sensor drive timing is correct or not can work it plays a decisive role. In this paper, based on the analysis of the timing of the CMOS image sensor IBIS5-B-1300, the design of the two register configuration program and two shutter and the system clock and ACD clock with a the FPGA embedded digital clock management unit (DCM) completed design. The experimental results show that the design of the drive timing to meet the drive requirements of the image sensor. The paper presents development of CMOS image sensor system based on chaos particle swarm.

\section{Chaos-based Particle Swarm Optimization}

Elementary particle swarm optimization precocious, make full use of the search feature of chaos optimization and particle swarm optimization, to dynamically shrink the search area, the chaotic particle swarm algorithm applied to structural reliability optimization theory, establish the structural system reliability constraints minimize structural quality optimization model is proposed based on the structure of the chaotic particle swarm algorithm reliability optimization design method [2]. The study results show that: the use of the reliability optimization design results significantly better than the results of the use of the particle swarm algorithm and best vector algorithm; the method is easy to implement, good stability, good engineering practical value and strong development capabilities.

Particle swarm optimization PSO Kennedy J and Eberhart R draw on the social behavior of flocks of birds and schools of fish predation process in 1995. The algorithm has a simple procedure, less control parameter optimization results with the initial value, and has a certain parallel features just a decade from the start of the study to the present, showing the powerful optimization features applied to the function optimization, neural networks, artificial intelligence, fuzzy system control and other fields. PSO as a more efficient parallel search algorithm is very suitable for solving the optimization problem in a complex environment has become a hotspot of evolutionary computation research.

Standard particle swarm algorithm showed a strong convergence, monotonic function, strictly convex function or univocal function at the initial time soon move closer to the optimal solution, but convergence is slow near optimal solution.

Chaos is the history of human sciences in the 20th century, following the theory of relativity and quantum theory after the third revolution, chaos is random state deterministic system evolution of the phenomenon, as a kind of non-linear systems, it is not by the random 
sexual external factors caused by deterministic rules is very sensitive to initial conditions lead to the long-term behavior of no fixed cycle. Chaotic movement within their own repeatedly traverse all the states, the initial value of the extremely weak condition changes will cause huge changes in the behavior of the system. Therefore, this article will be based on the improvement in the standard PSO, chaotic thinking is introduced into the particle swarm algorithm avoids the shortcomings easy to fall into local optimum value greatly improve the performance of the particle swarm algorithm optimization, as is shown by equation1.

$$
\begin{aligned}
\frac{\partial W}{\partial P} & =\left(\begin{array}{llllll}
\frac{\partial W_{x}}{\partial a_{1}} & \frac{\partial W_{x}}{\partial a_{2}} & \frac{\partial W_{x}}{\partial d_{1}} & \frac{\partial W_{x}}{\partial a_{3}} & \frac{\partial W_{x}}{\partial a_{4}} & \frac{\partial W_{x}}{\partial d_{2}} \\
\frac{\partial W_{y}}{\partial a_{1}} & \frac{\partial W_{y}}{\partial a_{2}} & \frac{\partial W_{y}}{\partial d_{1}} & \frac{\partial W_{y}}{\partial a_{3}} & \frac{\partial W_{y}}{\partial a_{4}} & \frac{\partial W_{y}}{\partial d_{2}}
\end{array}\right) \\
& =\left(\begin{array}{llllll}
x & 0 & y & 0 & 1 & 0 \\
0 & x & 0 & y & 0 & 1
\end{array}\right)
\end{aligned}
$$

PSO is simple and easy to achieve, are increasingly used in function optimization, network training, classification God mode, and the fields of application of the traditional optimization algorithms PSO algorithm there own limitations and defects, particle swarm optimization algorithm is easily fall into local extreme point, unable to get global optimal solution [3]. Algorithms at run-time parameters of the algorithm design, by the choice of the number of particles or inappropriate reasons.

Chaos is a general non-linear phenomena, their behavior is complex and similar random, but the exquisite inherent regularity. Periodicity of chaos, and chaos variables to optimize search more superior than reckless and disorderly random search, it avoids evolutionary algorithm into a local optimum drawback. Chaos has unique properties as follows: (1) the randomness that chaos has a messy performance similar to the random variable. (2) Periodicity of chaos can not duplicate after all the state of the certain range, (3) the regularity. Chaos is generated by the function determined by the relationship.

Hybrid optimization algorithm based on chaos theory improved particle swarm, despite the improved particle swarm optimization algorithm than the standard particle swarm optimization algorithm has been greatly improved, but due to the randomness of the initialization particles, some of the particle's position and its pestles to the $g$ best of the group, these particles is not zero because of its speed and inertia factor away from the optimal position and cause the algorithm does not converge close to zero when the speed is getting smaller and smaller, the diversity of the population is on the slows low to disappear, appear inert particles, as the iterative process proceeds, other particles will soon gathered these inert particles near and stop moving particle stagnation phenomenon, leading to premature convergence of the algorithm, the impact of the convergence of the algorithm.

$$
\frac{R^{n k} u^{(0)}}{\lambda_{1}^{n k}}=\sum_{i=1}^{N} \alpha_{i} \frac{\lambda_{i}^{n k}}{\lambda_{1}^{n k}}+\sum_{i=N+1}^{M} \alpha_{i} \frac{\lambda_{i}^{n k}}{\lambda_{1}^{n k}} e_{i}
$$

PSO is a new evolutionary computation method; the algorithm is simple, with fewer parameters to optimize performance better. In this paper, based on improvements in the standard PSO algorithm, the chaos theory to introduce particle swarm optimization, chaotic pseudo randomness to guide the particles of the initial value sensitivity and periodicity search, increase the diversity of the population and the particle search periodicity lose search capabilities speed reduced particle continue search capabilities [4]. Matlab software programming, the iterative process is given in an interactive interface; the computational 
results show that optimize performance has been greatly improved, and is a very effective optimization algorithm.

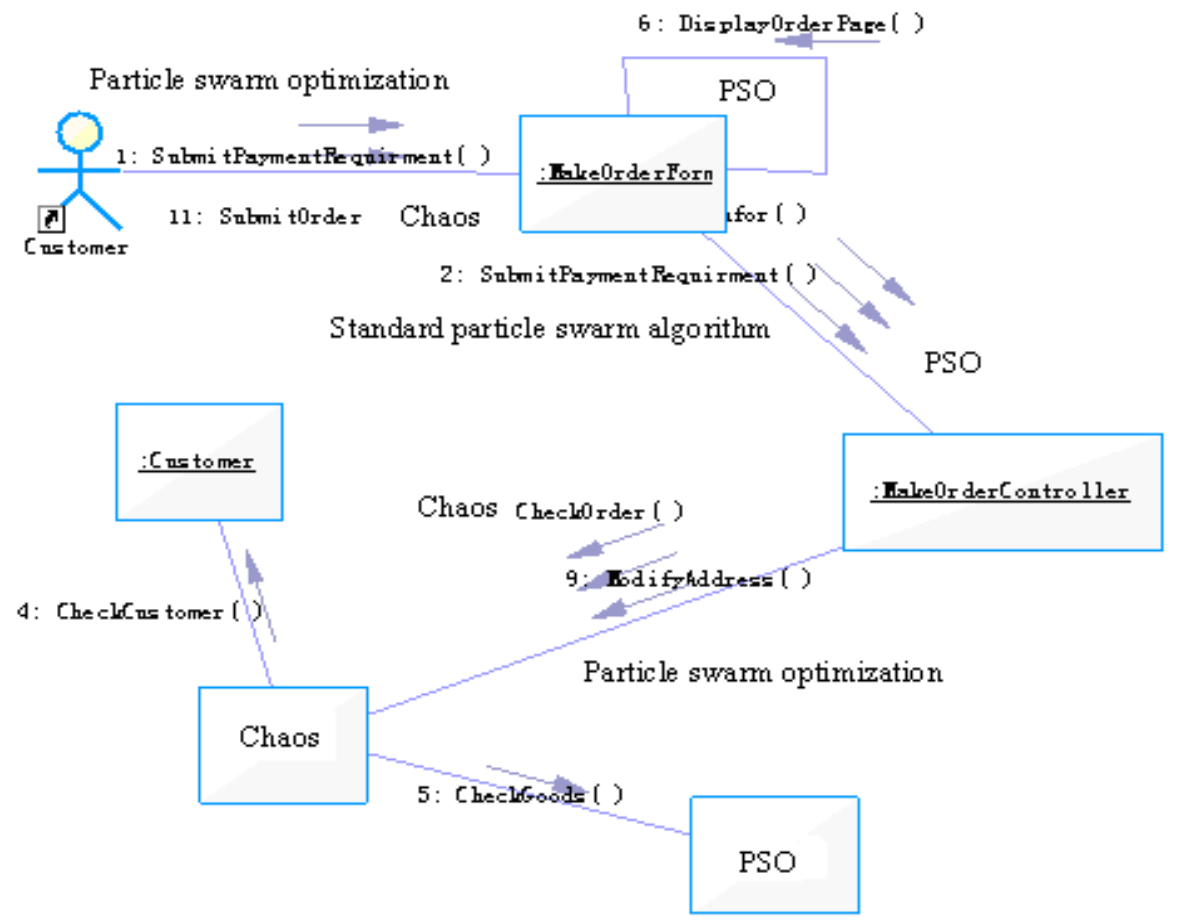

Figure 1. Chaos-based particle swarm optimization diagram

Program has a simple system parameters control characteristics, was quickly applied to the neural network training, Marketing Department identification, evolution and function of the digital circuit optimization, however, is similar to the genetic algorithm optimization algorithm, particle swarm algorithm also exists premature convergence and computing the amount of the larger short comings. chaos is a general non-linear phenomena, their behavior is complex and similar random, but its fine inherent regularity and periodicity of chaos in the optimization of the field can be characterized as a search processing an optimized mechanism to avoid falling into the local minimum chaotic particle swarm optimization algorithm also attracted the attention of the majority of scholars on the basis of the extensive literature paper summarizes the method and its research progress, and then by iteration to find the optimal solution in each iteration, the particle by tracking two "values" extremely renew itself: to find the optimal location of the particle itself, called pbest.

Currently, no chaos particle swarm optimization in structural reliability to optimize the design of the study reported. This author chaotic particle swarm optimization applied to structural reliability optimization design theory, minimize structural quality optimization model in structural system reliability constraints is proposed based on chaos particle swarm optimization the structural reliability optimization design method [5]. Finally, through the frame structure example, it is to verify the reliability of optimization theory guiding significance of the design methods and engineering practical value.

Particle Swarm Optimization PSO algorithm, each solution of the optimization problem is abstract without the mass and volume of the particle, and extends to the D-dimensional space. Located particle groups constituted by the $\mathrm{m}$ particles, the particle $\mathrm{i}(\mathrm{i}=1,2, \ldots, \mathrm{m})$ in $\mathrm{D}$ - 
dimensional space, the position and velocity, respectively, expressed as vector $\mathrm{zi}=(\mathrm{zi} 1$, each $\mathrm{zi} 2, \ldots, \mathrm{ZID}, \mathrm{ziD})$ and vector vi $=(\mathrm{vi} 1, \mathrm{vi} 2, \ldots, \mathrm{vid}, \ldots, \mathrm{viD})$, a particle has a fitness function fitness (zi) to calculate the the zi current fitness value, and thus measure the pros and cons of the position of the particle $. \mathrm{pi}=(\mathrm{pi} 1, \mathrm{pi} 2, \ldots, \mathrm{pid}, \ldots, \mathrm{piD})$ to search the optimal location for the particles so far, $\mathrm{PG}=(\mathrm{PG} 1, \mathrm{PG} 2, \ldots, \mathrm{PGD}, \ldots, \mathrm{PGD})$ as an integer.

Chaos particle swarm optimization algorithm, the conventional PSO optimization algorithm is not necessarily a study has achieved certain results, but there are still some shortcomings to be further studied: great or control inappropriate choice or traverse the interval when the search point search results is difficult to achieve the parameters of its control strategy selected is inappropriate, or close to the optimal solution, or algorithm may take very long, as is shown by equation 3 .

$$
\bar{R}_{i}(k)=\frac{1}{k} \sum_{j=1}^{k} R_{i}(j)=\frac{1}{k}\left[\sum_{j=1}^{k-1} R_{i}(j)+R_{i}(k)\right]
$$

Through a period of time will cause huge changes in the output. Chaos rich spatial and temporal dynamics, especially chaotic periodicity feature, it can be used as a search process to avoid falling into a local minimum optimization mechanism usually based on chaotic dynamical search process is divided into two stages as follows: First, based the of deterministic iterative traversal track on the entire solution space expedition that meet certain termination condition found in the search process state (Best So Far)close to the optimal solution of the problematic.

This paper introduces the idea of the chaos optimization particle swarm optimization algorithm, given the chaotic particle swarm optimization. The basic idea is to first chaotic optimization optimal particle groups of particles, and then chaos optimization results of random replacement of a particle in the particle population [6]. This treatment improved particle swarm optimization algorithm to get rid of the ability of local extreme point, to improve the speed and accuracy of the convergence of the algorithm. The simulation results show that the convergence of the chaotic particle swarm optimization algorithm outperforms the particle swarm optimization.

Particle Swarm Optimization stochastic optimization algorithms based on swarm intelligence, the basic idea is generated by the cooperation and competition between the population particle swarm intelligence guidance refine your search, simple principles and mechanisms, while maintaining a deep evolutionary algorithm group's smart background, and have a good optimizing performance. Therefore, PSO algorithm has been widely applied to the function optimization, multi-objective planning, neural network training, fuzzy system control and other fields. The fashion of traditional PSO algorithm to optimize complex functions save a lot of shortcomings, such as poor local search capability, search accuracy is not high, easy to fall into local optimum search late Yi shock. The researchers are from different points of view on the traditional PSO algorithm.

Chaos optimization idea is introduced into the particle swarm optimization algorithm to form a chaotic particle swarm optimization. This algorithm combines the periodicity characteristics of the chaotic initial value sensitivity and chaotic variables, and to improve the ability of the particle swarm optimization algorithm to get rid of local extreme point, to improve the accuracy of the convergence of the algorithm. Chaotic particle swarm optimization algorithm is applied to power system economic allocation problem solving [7]. The examples show that the algorithm is compared with other algorithms with the high quality of the optimal solution, convergence and good benefits. Due to the versatility of 
chaotic particle swarm algorithm, the algorithm is expected to be applied to a wider range of optimization problems.

\section{Research of CMOS Image Sensor System}

The expansion of the scope of the image sensor applications, as well as the flexibility and performance enhancements in the majority of applications in digital imaging technology has replaced the old film-based imaging technology. Current image sensor innovation is to shorten the exposure time at the same time enhancing the sensitivity, pixel manner, it becomes smaller and smaller, the end result is that the image sensor simply can be imaged with a small amount of light in the spectral; the manufacturers are also larger laser sensor development, applied to the optical range. However, in order to ensure that the main position of the image sensor, you need to understand all the limitations of the manufacturing image sensor manufacturers need to pass hardware and software to look at the difference with other competitors.

CMOS image sensors can be divided into passive pixel sensor (PPS), and active-active pixel sensor (APS).The photogene rated charge generated photodiode APS is divided into type, grating and logarithmic response type now proposed DPS (digital pixel sensor) concept.

The image sensor is the use of the principle of a photoelectric conversion to an apparatus that converts the image data into a series of electronic signals. Contact Image Sensor CIS appeared in the late 1980s, a new type of image sensor based on CMOS technology based photoelectric scanning device [8]. CIS compared to CCD has the advantages of small size, high speed, low cost, simple installation and commissioning. However, both the CIS and CCD must take into account the impact of the interference signal, sensor deviation factor of the image signal, only corrects for these effects, in order to faithfully reproduce the image information.

In order to improve the image quality of a CMOS image sensor, through the analysis of the image the main source of noise and image distortion, the paper proposes a novel CMOS active pixel image sensor. The CMOS image sensor uses a 4T active pixel, greatly improving the sensitivity of the image sensor. Through the image preprocessing functions integrated in the sensor, to improve the quality of the image play a very good effect, as is shown by Figure2.

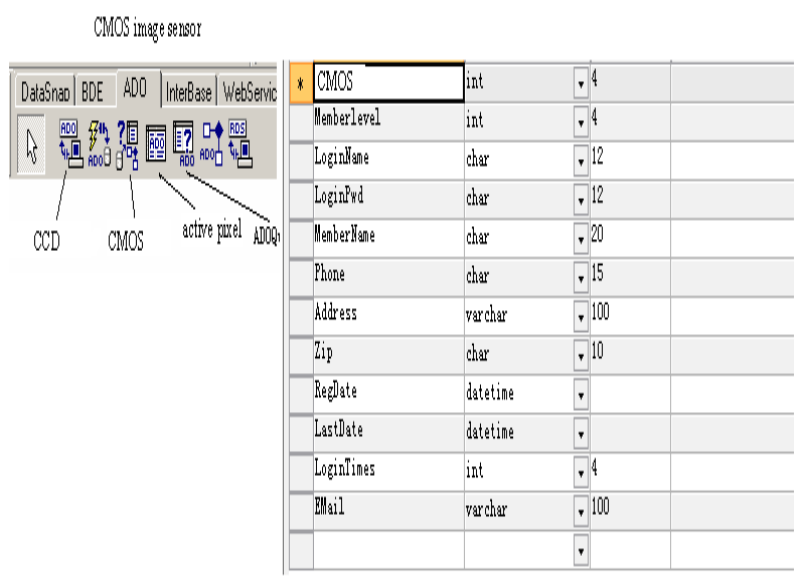

Figure 2. CMOS image sensor system 
After sampling of the image, the electronic signal is proportional to the image gray value of the photoelectric sensor output analog; therefore, it must be put into a digital signal, in order to input into the computer for data processing. This kind of the image information of the actual output of the analog the quantitative replace discrete digital conversion process is called quantization. Uniform quantization technology is $\mathrm{Vp}$ and $\mathrm{Vd}$ the difference $\mathrm{Vp}-\mathrm{Vd}$ intervals take $\mathrm{K}$ levels, the normal quantization level $\mathrm{K}=2 \mathrm{M}$, if the choice of the eight $\mathrm{A} / \mathrm{D}$ converter, then $\mathrm{m}=8$, and $\mathrm{K}=256$ levels. Actual output range between $\mathrm{Ri}$ and $\mathrm{Vi}$ take quantized values $\mathrm{Vi}$, to take $\mathrm{Vi}+1$ range between $\mathrm{Ri}$ and $\mathrm{Vi}+1$. Below is calculated by the mean square error technique, quantization decision level values when the quantization error is the most hours.

CMOS image sensor in the 1980s, the CMOS process manufacturing technology was not high, so that the sensor noise in the application and commercialization of the process has been very slow [9]. With the continuous improvement of the process, so far, the range of applications for CMOS image sensor expanding it comes to digital products, communications, industrial, medical and other fields. Compared with CCD, CMOS image sensors with small size, low power consumption, and low cost [10]. The Cypress CMOS image sensor IBIS5-B-1300 is a high performance, large dynamic range of the image sensor. The correct drive timing signal for the normal work of the image sensor on the image sensor IBIS5-B-1300, given the drive timing and simulation results using VHDL language design.

$$
\begin{aligned}
\Delta u^{T} S \Delta u & \rightarrow u^{T} P^{T} S P u \\
\Delta u & =P u \\
\Delta u(i) & =u(i-1)-u(i)
\end{aligned}
$$

CMOS image sensor for the imaging unit, design a high-performance image sensor imaging system. Concrete realization of the hardware part of the system, the drive and control the FPGA using Verilog HDL, low dropout regulator to achieve stable supply of CMOS image sensors and devices, PCB multilayer achieve. Especially with a modular design, the system has the characteristics of a small, high stability and flexibility.

In order to obtain a good image quality, it is the need for the original image data acquisition processing. Usually, the image preprocessing is completed in the coprocessor. Recently, with the development of SoC technology can be integrated in a CMOS sensor image pre-processing functions. This shows the advantage of the CMOS image sensor [11]. The image preprocessing main including defect correction, remove FPN noise, and it is a series of processing of the color difference and the difference in image sharpening, aperture correction, Gamma correction. Achieved through digital image processing algorithms to the image to achieve the above-described pretreatment process, the hardware platform can be integrated in the image processing circuit in the SoC, ASIC image processing chip, or general-purpose DSP chip.

CMOS image sensor has an amplifier in each pixel position, which it converted into discrete charge signal packets in the case of low bandwidth voltage output, but also requires only reset in the frame rate. One of the advantages of a CMOS image sensor is that it has a low bandwidth, and increases the signal-to-noise ratio. Due to manufacturing process constraints of the amplifier, the earlier CMOS image sensors can not be placed within the pixel position. This is referred to as PPS technology, the noise performance is not ideal, but also led to various interferences on the CMOS image sensor is as follows formula 5 [12]. 


$$
\overline{I_{k}}=\frac{\sum_{x \in \Omega_{i}}\left[I_{k}\left(X^{\prime}\right)\right]}{L \cdot L}, \overline{I_{k-1}}=\frac{\sum_{x \in \Omega_{i}}\left[I_{k-1}(X)\right]}{L \cdot L}
$$

CMOS image sensor image quality in low light has been as good as CCD, thereby improving image quality CMOS image sensor developed focus. Toshiba the novel structure of the buried photodiode, reducing the leakage current, and also ensures that the non-charged residue is completely read out at low pressure, to achieve a high quality image equivalent to the CCD image pickup device.

The unique DRSCAN (Dot the Sequential Readout System with the Current Amplified of Signal the Output Noise Reduction Circuit) technology is in the point-by-point sequential read out of each pixel signal and a noise component at the same time, the elimination of transistor properties in the same circuit unevenness caused by the fixed pattern noise this is the progressive elimination of difficult to do. In order to eliminate fixed pattern noise caused by the dark current, but also learn the CCD HAD (Whole accumulation diode) structure [13]. The whole accumulation layer is formed on the sensor surface, thereby suppressing the dark current caused by the non-incident. Two fixed pattern noise reduced, so that $\mathrm{S} / \mathrm{N}$ ratio increased 25 times, to achieve a high quality of the CMOS image sensor. HAD structure of a pixel structure of the L-shaped gate, so that almost all of the electrons are completely transferred to achieve a non-smear image signal output, as is shown by equation6.

$$
F(x, y)=\frac{\sum_{i} w(d(x, y)) I_{i}(x, y)}{\sum_{i} w(d(x, y))}
$$

Three categories of development of CMOS image sensors, CMOS-PPS CMOS-APS, and CMOS-DPS (Digital Pixel sensor), and CMOS-DPS the last two years developed [15]. December 2001 the Kodak, coda, Hewlett-Packard, Agilent Technologies and Stanford University and the University of California, and other standard digital $0.18 \mathrm{~m}$ CMOS process development successful high frame rate $(10000 / \mathrm{sec})$ CMOS digital pixel sensor. Performance parameters: the number of pixels 352,288; chip size 5mm; transistor 3,800,000; readout structure for the $64 \mathrm{bit}(167 \mathrm{MHz})$; maximum output data rate greater than $1.33 \mathrm{~GB} / \mathrm{s}$; maximum continuous frame rate greater than $10000 / \mathrm{sec}$; the maximum continuous pixel rate to greater than 1Gpixels / s; pixel size of $9.4 \mathrm{~m}(\mathrm{H}) 9.4 \mathrm{~m}(\mathrm{~V})$; photo detector type $\mathrm{n}$ MOS photoelectric gate; the number of transistors for each pixel is 37 ; the devices single pixel by the photodiode, the analog-to-digital converter (ADC), a digital memory and correlated double sampling (CDS) circuit and the like.

In order to obtain a good image quality, it is the need for the original image data acquisition processing. Usually, the image preprocessing is completed in the coprocessor. Recently, with the development of Sac technology can be integrated in a CMOS sensor image pre-processing functions. This shows the advantage of the CMOS image sensor. The image preprocessing main including defect correction, remove FPN noise and a series of processing of the color difference, it is the difference in image sharpening, aperture correction, Gamma correction. 


\section{Development of CMOS Image Sensor System based on Chaos Particle Swarm}

Particle Swarm Optimization in solving complex function, there is a slow convergence, high accuracy, easy to fall into local minima and other issues. To this end, an adaptive chaotic particle swarm optimization algorithm. Particle Swarm Optimization introduces chaos variables, the chaotic search algorithm premature convergence, while introducing a nonlinear decreasing inertia weight. The experimental results show that the algorithm has a fast convergence rate and high convergence precision, and can effectively avoid the premature convergence problem.

Introduction of mutation operators in the improved algorithm, sometimes variation in the mutation process is invalid, but increased the amount of computation. Has more superior performance, in order to make chaotic particle swarm optimization, how to select the search starting point, how narrow the search limited local search space, how about, such as design, how to design a good termination criterion, how to select the appropriate initial control parameters and their control, such as the system strategy, which is how to design a more effective algorithm is the key to further study in the later work.

CMOS image sensor is a new sensor developed in recent years, compared to solid-state CCD (Charged Coupled Device) sensor, which has a small size, light weight, high integration, and low power consumption, low cost, convenient programming, easy to control the speed advantages. Especially in recent years, with the sub-micron and deep sub-micron process technology development and the continuous improvement of the device structure, CMOS image sensor, image quality is close to or reaches the image quality of a CCD image sensor. Based on these characteristics, the CMOS image sensor suitable for high frame rate image acquisition system. LUPA-300 high-speed CMOS image sensor is briefly introduced, and based on this sensor, high frame rate CMOS imaging unit, image storage unit, image data acquisition unit and system software is designed to establish a high-speed CMOS image acquisition system, as is shown by equation 7 .

$$
\sigma\|(V(k, t))\|=\sqrt{\frac{1}{n} \sum_{i=0}^{n-1}\left(\|\overline{V(k, t)}\|-\left\|V_{i}(k, t)\right\|\right)^{2}}
$$

A chaotic variable within a certain range has the following characteristics: randomness, its performance with random variable messy; periodicity, that it can not repeat their way through all the states within the space; regularity, the variable is determined by the iteration the equation export. Chaos optimization method is a novel optimization method, which uses unique periodicity of the chaotic system to achieve the global optimum, and it does not require the objective function continuity and differentiability nature.

Chaos Particle Swarm Optimization Chaos particle swarm algorithm is an irregular state of motion, you can generate random behavior in deterministic nonlinear systems without any additional random factor. Chaotic system has a special law of motion, mainly for the randomness, regularity and periodicity. The chaotic state general the randomness movement is by deterministic equations. In this paper, the image acquisition system based on high-speed CMOS image sensor with easy control, safe and reliable, stable performance. The system can be extended upon request does not change the premise of the system hardware, image less demanding situations can increase the image compression unit to increase the flexibility of the system, so that a broader range of applications.

Host application using VC + development, VC ++ MFC framework to write a multithreaded image acquisition and processing procedures. User by calling the correlation 
function, you can complete the configuration of the data acquisition card. Application through the PCI bus to receive the original image data of the SRAM memory, until after receiving the data, the application can be performed on the image data with a simple processing and analysis, and the image is displayed, while the image data stored in the hard disk, and the completion of image data the acquisition, display and storage. The application can also collect images for subsequent processing.

Chaos optimization thinking particle swarm optimization algorithm is given the chaotic particle swarm optimization algorithm. The basic idea is to each particle swarm in the evolutionary process, the optimal particle particle groups' chaos optimization, and then randomly selects a particle from the particle population to replace with chaos optimization results. This treatment makes the particle swarm optimization algorithm to get rid of local optimum capacity improved to improve the speed and accuracy of the convergence of the algorithm. Simulation results show that chaotic particle swarm optimization convergence of the algorithm performance is significantly better than the particle swarm optimization algorithm, and also better than the hybrid particle swarm optimization algorithm and particle swarm optimization with Gaussian mutation particle swarm optimization algorithm is slightly better than with the inertia factor, as is shown by Figure3.

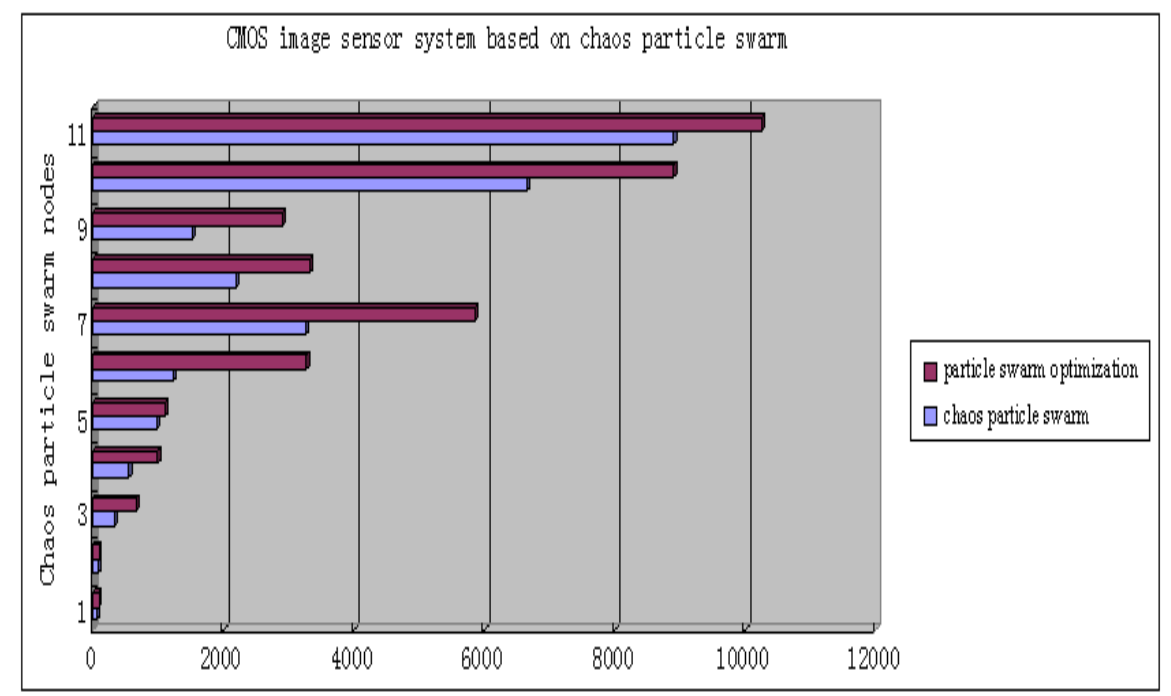

Figure 3. CMOS image sensor system based on chaos particle swarm

The paper presents development of CMOS image sensor system based on chaos particle swarm. As can be seen from the graph, chaotic particle swarm optimization algorithm convergence performance is better than that of particle swarm optimization algorithm convergence performance. Figure 3 is the particle swarm optimization algorithm and chaotic particle swarm optimization algorithm numerical simulation results, the map can be seen, chaotic particle swarm optimization algorithm for solving function the result is better than that of particle swarm optimization algorithm for solving results (509 independently run average). The other function is done by a great number of computer simulation results also prove this point, due to space limitations, here no longer give results. 


\section{Conclusions}

These algorithms from different aspects of particle swarm optimization algorithm were improved in different degree, improve the speed of convergence and accuracy, but the effect is not very ideal. Chaos is a natural phenomenon of nonlinear. Chaotic variables are seemingly random change process which contains internal regularity, using chaos randomness, periodicity and regularity can be optimized for search. A CMOS imaging unit mainly consists of a CMOS image sensor and control chip FPGA, it is the system imaging components, to capture the speed of the moving object image, and the circuit output is a digital image data. Image storage unit is mainly composed of a static memory SRAM, responsible for the imaging unit outputs the image data in real time for computer data acquisition, storage, processing of the image data. The image data acquisition unit is mainly composed of data acquisition card and data acquisition interface circuit, the completion of image storage unit stores the image data collection work. The system software using VC++ development, completion of the hardware circuit and the control to the acquisition unit to the image data processing and display, reproducible imaging unit captured image.

\section{References}

[1] W. Wei and B. Zhou, "Features Detection Based on a Variational Model in Sensornets", JDCTA, vol. 4, no. 7, (2010), pp. 115-127.

[2] J. S. Prathipa and T. R. Lakshminarayanan, "PSNR Based Clustering and Indexing for Fast Access", Journal of Theoretical and Applied Information Technology, vol. 38, no. 2, (2012), pp. 170-176.

[3] M. Anbar and D. P. Vidyarthi, "Router CPU Time Management using Particle Swarm Optimization in Cellular IP Networks", IJACT, vol. 1, no. 2, (2009), pp. 48-55.

[4] J. Gao, Z. Lei, Z. Wang and H. Li, "Sequence Image Match Based on Salient Point Invariants Moments", JDCTA, vol. 6, no. 11, (2012), pp. 124-130.

[5] L. Tian, X. Liu, J. Li and X. Guo, "Image Preprocessing of CMOS Image Acquisition System Based on FPGA”, JDCTA, vol. 6, no. 20, (2012), pp. 130-139.

[6] J. -q. Xiong, J. -h. Huang and Q. Liao, "Simulation Analysis of Controlling Buffeting Noise Based on Hybrid Particle Swarm Algorithm”, JCIT, vol. 7, no. 23, (2012), pp. 753-761.

[7] H. Xue and W. -b. Li, "Implementation of Chaos Synchronization on FPGA", AISS, vol. 4, no. 6, (2012), pp. 42-51.

[8] Y. Liping, S. Kai and W. Chao, "Design and Realization of Forest Fire Monitoring System Based on Image Analysis", JDCTA, vol. 5, no. 12, (2011), pp. 452-458.

[9] S. Samundeeswari and M. Thiyagarajan, "Knowledge Based Approach for Alignment Problems", Journal of Theoretical and Applied Information Technology, vol. 27, no. 1, (2011), pp. 134-142.

[10] J. Lv and Y. Zhang, "Teaching evaluation method based on least squares support vector machine and chaos particle swarm optimization algorithm”, JDCTA, vol. 6, no. 11, (2012), pp. 343-351.

[11] Y. Bai and X. Shi, "A Novel Neural Network Wave-Front Predictor Combined with Parallel Chaotic PSO Algorithm”, JDCTA, vol. 6, no. 23, (2012), pp. 430-437.

[12] C. Shi, Y. Wang and H. Zhang, "Faults Diagnosis based on Support Vector Machines and Particle Swarm Optimization", IJACT, vol. 3, no. 5, (2011), pp. 70-79.

[13] M. Gopinath and S. Ramareddy, "Simulation of Closed Loop Contrlled Bridgeless PFC Boost Converter", Journal of Theoretical and Applied Information Technology, vol. 10, no. 2, (2009), pp. 180-191. 


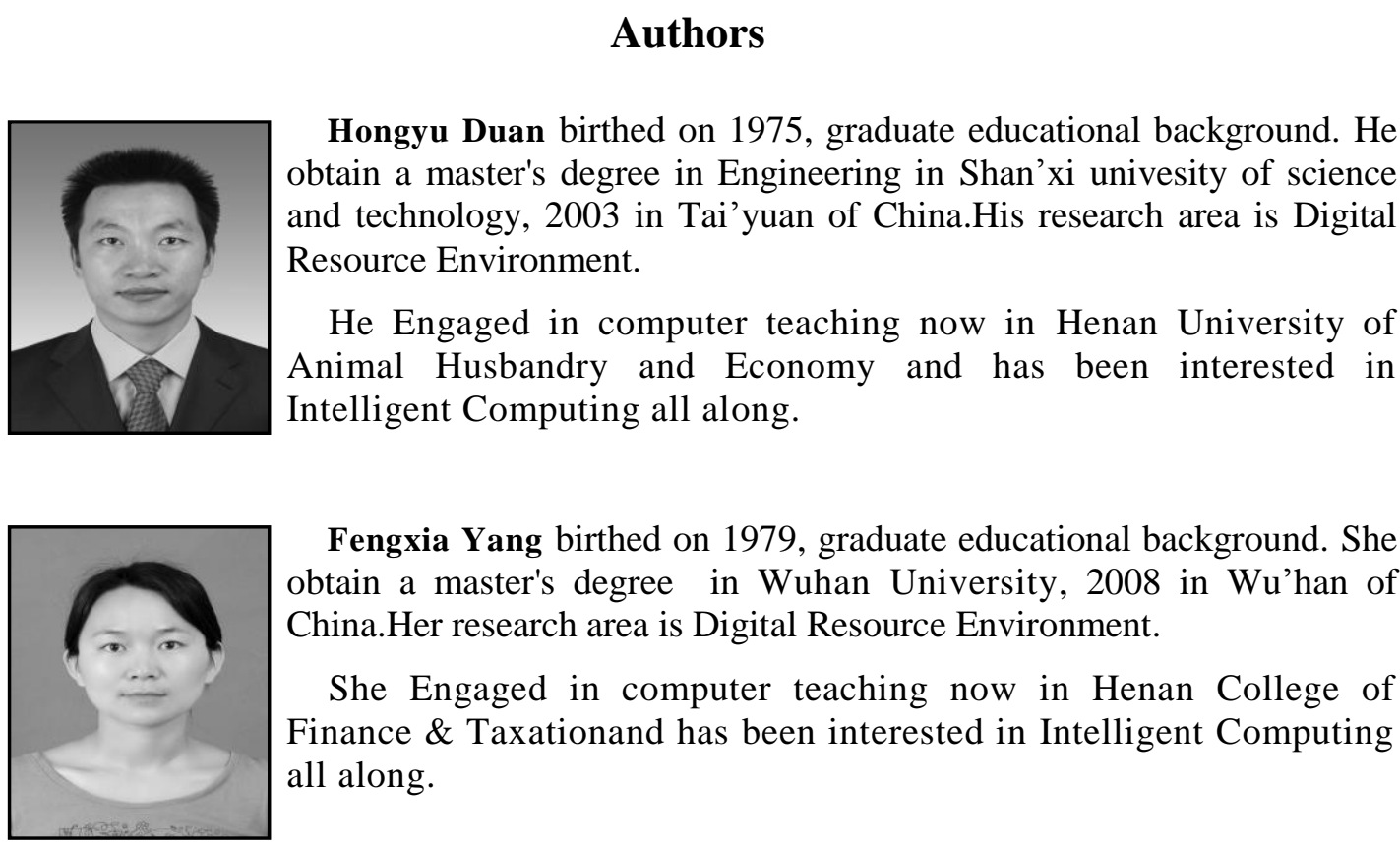

